\title{
Critérios Prognósticos de Sucesso e Recorrência na Ablação Circunferencial para Tratamento da Fibrilação Atrial
}

Prognostic Criteria of Success and Recurrence in Circumferential Ablation for the Treatment of Atrial Fibrillation

\author{
Washington Maciel, Eduardo Andréa, Nilson Araújo, Hecio Carvalho, Luiz Gustavo Belo, Leonardo Siqueira, Claudio \\ Munhoz, Rodrigo Cosenza, Fabiana Mitidieri, Jacob Atié \\ Clínica São Vicente, Instituto Estadual de Cardiologia Aloysio de Castro e Universidade Federal do Rio de Janeiro - Rio de Janeiro, RJ
}

\section{Resumo}

Objetivos: Analisar o sucesso da ablação circunferencial da fibrilação atrial e investigar possíveis preditores, clínicos e eletroanatômicos, de recorrência da arritmia.

Métodos: Foram analisados 104 pacientes consecutivos, submetidos à ablação circunferencial para tratamento de fibrilação atrial paroxística/persistente, sem cardiopatia estrutural e refratários a pelo menos duas drogas antiarrítmicas. Eram do sexo masculino 72 pacientes e a idade média do grupo foi de 58,6 + 10,9 anos. O procedimento consistiu em punção transeptal única e mapeamento tridimensional, com o sistema CARTO $^{\circledR}$ para a aquisição de pontos no átrio esquerdo e veias pulmonares. As aplicações de radiofreqüência foram realizadas ao redor dos óstios das veias pulmonares, até a redução $\geq \mathbf{8 0} \%$ da amplitude dos potenciais atriais. Uma linha adicional foi criada no istmo mitral e outra no istmo cavotricuspídeo. Foram analisadas: volume total do átrio esquerdo, área ablacionada ao redor das veias pulmonares e a presença ou não de falhas na linha de ablação (linha completa ou incompleta). Foi considerada linha completa quando a distância entre dois pontos contíguos de aplicação de radiofreqüência foi inferior a $10 \mathrm{~mm}$.

Resultados: Em acompanhamento médio de 18 meses, 87 pacientes estavam em ritmo sinusal (84\%) e 17 pacientes apresentaram recidiva (16\%). Na análise multivariada, somente o volume atrial esquerdo $(p<0,0001)$ e a ablação completa $(p<0,05)$ foram preditores independentes de recorrência.

Conclusão: Os resultados sugerem que o volume atrial esquerdo e a presença de ablação completa são preditores de recorrência da fibrilação atrial.

Palavras-chave: Fibrilação atrial, mapeamento eletroanatômico, ablação por cateter.

\section{Summary}

Objectives: To analyze the success of circumferential ablation on atrial fibrillation and investigate possible clinical and electro-anatomical predictors of the recurrence of cardiac arrhythmia.

Methods: Analysis was done of 104 consecutive patients, free of structural heart disease and refractory to at least 2 antiarrhythmic drugs, submitted to circumferential ablation for treatment of paroxysmal/persistent atrial fibrillation. Were males 72 of the patients and the average age of the group was $58.6+10.9$. The procedure consisted in a single transeptal puncture and three-dimensional mapping using the CARTO ${ }^{\circledR}$ system. Images of the left atrium and pulmonary veins were reconstructed. Radiofrequency applications were performed around the pulmonary vein ostia, up to a $\geq 80 \%$ reduction in atrial potential amplitude. An additional mitral isthmus line was created, as well as one in the cavotricuspid isthmus. Total left-atrial volume, area ablated around the pulmonary veins, and the presence or absence of flaws in the ablation line (complete or incomplete line) were analyzed. A line was considered complete when the distance between two contiguous radiofrequency application points was less than $10 \mathrm{~mm}$.

Results: After an average follow-up period of 18 months, 87 patients were in sinus rhythm (84\%), while 17 patients presented recidivism (16\%). In the multivariate analysis only left-atrial volume $(p<0.0001)$ and complete ablation $(p<0.05)$ were independent predictors of success.

Conclusion: These data suggest that the left-atrial volume and the presence of complete ablation are predictors of recurrence.

Key words: Atrial fibrillation, electroanatomical mapping, catheter ablation.

Correspondência: Washington Andrade Maciel •

R. Barão de Lucena, 76/501 - 22260-020 - Rio de Janeiro, RJ

E-mail:wmaciel@cardiol.br

Artigo recebido em 03/01/06; revisado recebido em 13/05/06; aceito em 01/06/06. 


\section{Introdução}

A fibrilação atrial é a arritmia sustentada mais freqüente na prática clínica, diagnosticada por clínicos e cardiologistas, presente em todos os ambulatórios, tanto mais prevalente quanto maior a faixa etária ${ }^{1-6}$

A manutenção do ritmo sinusal com drogas antiarrítmicas e cardioversões sucessivas, como apresentada no estudo AFFIRM e nos subestudos subseqüentes ${ }^{7-9}$ demonstrou ser uma estratégia que não beneficiou os pacientes, no que concerne à mortalidade. Na verdade o AFFIRM demonstrou aumento da mortalidade no grupo de controle do ritmo, quando foi comparada com a simples manutenção da freqüência ventricular, com $p=0,08$.

O tratamento não farmacológico, nas diversas abordagens propostas, apresenta resultados satisfatórios, isto é, manutenção do ritmo sinusal, com sucesso variando entre $60 \%$ e $90 \%{ }^{10}$. Essa ampla variação é dependente da população estudada, do método empregado, dos critérios de sucesso e da experiência da equipe, entre outros.

A identificação de critérios possivelmente preditores de sucesso e insucesso, previamente ao procedimento e durante o mesmo, pode ser útil para definir a extensão da ablação, a necessidade de linhas de radiofreqüência adicionais ou abordagem de potenciais atriais anormais.

Outro aspecto prático, baseado no conhecimento dos preditores de sucesso e recorrência é a avaliação do possível resultado da ablação por radiofreqüência da fibrilação atrial. Assim, é possível criar uma expectativa quanto aos resultados no longo prazo, realística, sem pessimismos desnecessários ou otimismos infundados.

Este estudo tem como objetivo analisar o sucesso e o insucesso dos pacientes submetidos à ablação por radiofreqüência da fibrilação atrial, após um único procedimento, e identificar possíveis preditores clínicos, eletrofisiológicos e eletroanatômicos de recorrência da arritmia através da análise dos mapas anatômicos construídos durante a ablação e avaliar as complicações do procedimento.

\section{Métodos}

Foram analisados 104 pacientes consecutivos, portadores de fibrilação atrial paroxística recorrente ou fibrilação atrial persistente, submetidos à ablação circunferencial das veias pulmonares, guiada pelo mapeamento eletroanatômico, utilizando-se o Sistema CARTO ${ }^{\circledR}$, de março de 2002 a julho de 2003.

A idade média do grupo estudado foi de 58,6 \pm 10,9 anos, variando entre 20 e 80 anos, sendo 72 pacientes do sexo masculino e 32 do sexo feminino.

Foram incluídos, após consentimento informado e esclarecido, pacientes com fibrilação atrial paroxística e persistente, há mais de um ano, sintomática, recorrente, documentada através de registros eletrocardiográficos, refratária a duas ou mais drogas antiarrítmicas, incluindo amiodarona.

Foram excluídos pacientes com impossibilidade de uso crônico de Warfarin, portadores de lesão orovalvar grave, insuficiência cardíaca ou comorbidades associadas, bem como pacientes com fração de ejeção $\leq$ a $35 \%$ e ou com trombo intracavitário.

Previamente ao procedimento os pacientes foram submetidos a angiotomografia ou angiorressonância do átrio esquerdo e veias pulmonares, para a definição da anatomia destas estruturas, tratamento com Warfarin por pelo menos 3 semanas, objetivando-se a manutenção do nível de INR ente 2 e 3, suspenso 7 dias antes do procedimento com introdução de enoxiparina $(1 \mathrm{mg} / \mathrm{kg})$ de 12/12 horas até 24 horas antes do procedimento e ecocardiograma transesofágico nas 24 a 48 horas que precederam o procedimento.

O procedimento foi realizado sob sedação consciente com emprego de baixas doses de Midazolan associado ou não ao Propofol.

Após a punção transeptal os pacientes receberam heparina não fracionada, em doses progressivas, objetivando-se a manutenção do tempo de coagulação ativado (TCA) $\geq$ a $250 \mathrm{seg}$.

O Mapeamento e a ablação - Após uma punção transeptal única inseriu-se então o cateter mapeador (NAVI-STAR ${ }^{\circledR}$ Cordis-Webster - ponta de $4 \mathrm{~mm}$ ), para a reconstrução espacial do átrio esquerdo e veias pulmonares. O limite entre a veia e o átrio foi definido pela súbita diminuição da impedância, associada ao aparecimento de significativo potencial atrial e à imagem fluoroscópica de "queda" do cateter dentro da câmara atrial.

Para o procedimento utilizado neste estudo interessou apenas o mapa anatômico, que define a estrutura espacial da câmara analisada, o átrio esquerdo, a válvula mitral e as veias pulmonares. Este mapa registra o átrio de forma monocromática e apenas distingue com outra cor as veias pulmonares e os pontos de aplicação de radiofreqüência (fig. 1).

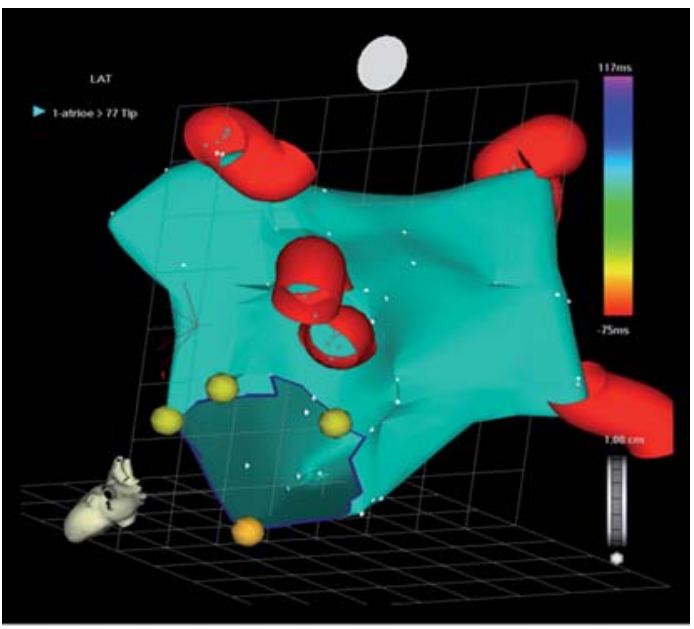

Fig. 1 - Reconstrução da Câmara Atrial Esquerda.

Visão posterior do átrio esquerdo com mapa anatômico. As veias estão marcadas em vermelho, a válvula mitral é vista como um "vazio" na imagem anatômica. 


\section{Artigo Original}

Após a reconstrução da anatomia atrial esquerda, o mesmo cateter mapeador foi utilizado para emissão de radiofreqüência. A programação da energia foi feita para ablação com controle de temperatura (máxima programada para $60^{\circ} \mathrm{C}$ ) e potência limitada a 50 W. O aparelho de radiofreqüência foi o Stokert ${ }^{\circledR}$ (Cordis-Webster). Considerou-se a aplicação de energia eficaz quando o potencial local apresentou diminuição $\geq 80 \% \mathrm{em}$ relação ao registrado antes da ablação; assim, este local foi marcado como um ponto de radiofreqüência, passando-se, imediatamente, a aplicar em região contígua. O tempo de liberação de energia de radiofreqüência para a obtenção da redução da amplitude do potencial varia, em média, entre 5 a 15 segundos, por ponto.

O objetivo do procedimento foi circundar as veias esquerdas e direitas, de uma forma única, não deixando intervalo $\geq 10 \mathrm{~mm}$ entre os pontos de radiofreqüência.

A avaliação da completude da linha de radiofreqüência foi, portanto, determinada pela medida da distância entre os pontos contíguos de radiofreqüência. Foi considerada linha incompleta quando se observou distância $\geq 10 \mathrm{~mm}$ entre pontos contíguos. Nos outros casos a linha de ablação foi considerada completa.

O intervalo de $10 \mathrm{~mm}$ foi assumido como valor a partir do qual uma linha de aplicação de radiofreqüência seria considerada incompleta, ou seja, com lacuna ou gap. Esse valor foi escolhido considerando-se que um cateter de ablação, com ponta de $4 \mathrm{~mm}$, quando libera energia de radiofreqüência com temperatura programada para $60^{\circ} \mathrm{C}$ e potência máxima de até $50 \mathrm{~W}$, em contato com o músculo cardíaco, produz uma lesão que pode variar entre 2 e 11 $\mathrm{mm}^{11,12}$. Portanto, uma distância superior a $10 \mathrm{~mm}$ entre pontos contíguos permite a existência de músculo não ablacionado e, assim sendo, haveria possíveis caminhos de passagem para impulsos elétricos, vindos das áreas de ablação circunferencial.

A medida da distância entre os pontos é fornecida pelo próprio sistema CARTO ${ }^{\circledR}$.

Alguns pacientes também apresentavam episódios de flutter atrial típico, documentado eletrocardiograficamente. Não foi induzido o flutter para avaliação do seu circuito, porém, em todos os pacientes foi criado bloqueio bidirecional da condução istmal.

A retirada das bainhas (duas venosas e uma arterial) só foi realizada após o procedimento, quando o tempo de coagulação ativado atingiu valores inferiores a $180 \mathrm{~s}$.

Foram analisadas as seguintes medidas nos mapas eletroanatômicos: o volume do átrio esquerdo, o volume da área ablacionada ao redor das veias direitas e esquerdas, a completude da aplicação de radiofreqüência circunferencial, o número de pontos de aplicação de energia e a relação entre o volume circunferencial de aplicação de radiofreqüência das veias esquerdas e direitas e o volume atrial esquerdo.

As variáveis clínicas analisadas foram: idade, sexo, presença de hipertensão arterial e doença arterial coronariana.

A presença de doença arterial coronariana foi definida pela demonstração, por coronariografia, de lesões significativas, necessitando tratamento clínico ou não farmacológico.
A hipertensão arterial foi definida pela história clínica e níveis tensionais $\geq 130 / 90 \mathrm{mmHg}$ em consulta anterior ao procedimento.

Duas variáveis ecocardiográficas foram analisadas: a presença de hipertrofia ventricular esquerda, definida como septo e parede posterior mais espessados que o limite máximo normal de 1,1 cm, e a fração de ejeção medida pela fórmula de Teichhoolz.

A medida do volume atrial esquerdo foi adquirida, automaticamente, quando finalizada a reconstrução da câmara atrial, pelo próprio Sistema CARTO ${ }^{\circledR}$

A ablação circunferencial das veias pulmonares tem forma aproximadamente circular, naturalmente irregular, dada a complexidade da anatomia atrial.

Para o cálculo da área de ablação circunferencial, uma vez que é aproximado a um círculo, a única medida que se faz necessária é a medida de seu raio. Neste trabalho, foi calculado um raio médio, por meio da média de segmentos que cortassem o átrio passando por seu centro geométrico.

Utilizou-se como diâmetro médio, a média dos quatro segmentos que interceptam a figura e, como raio médio, a metade desse diâmetro. A área da figura passa a ser então: Área $=\pi 1 / 4(a+b+c+d)^{2}$ onde $a, b$, c e d são os comprimentos dos segmentos de reta que interceptam o átrio em seu centro geométrico.

Este sistema de mapeamento permite a visualização da área de ablação com e sem as veias, tornando mais fácil a medição do raio médio. Como a imagem pode ser analisada sob vários ângulos, procurou-se a melhor imagem da área de ablação em relação à superfície atrial, conforme a figura 2 .

Os pacientes estudados foram liberados do hospital 24 horas após o procedimento eletrofisiológico, com prescrição de Warfarin, Enoxiparina e o mesmo antiarrítmico que vinha sendo usado antes da ablação, embora este fosse considerado ineficaz devido à recorrência pré-ablação.

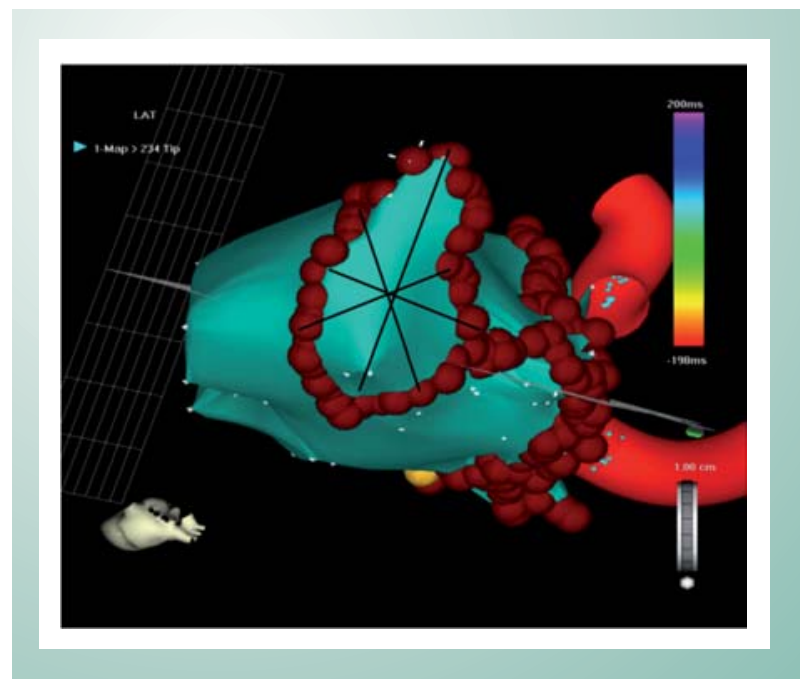

Fig. 2 - Medidas para a análise da área de ablação.

A área da figura é calculada pela seguinte fórmula: $A=\pi 1 / 4(a+b+c+d)^{2}$, onde $a, b$, c e d são os comprimentos dos segmentos de reta que interceptam $o$ átrio em seu centro geométrico. 
O acompanhamento dos pacientes fez-se através de consultas médicas após 15 dias, 30 dias, 3 e 6 meses e consultas sucessivas com intervalo $\leq$ a 6 meses.

Considerou-se insucesso a recorrência da fibrilação atrial paroxística ou persistente após 45 dias da ablação e os sintomas sugestivos de arritmia, independentemente da confirmação eletrocardiográfica de fibrilação atrial, que não respondiam à introdução de antiarrítmicos.

Foi considerada ablação bem-sucedida, após 45 dias, a ausência de fibrilação atrial, documentada eletrocardiograficamente, ou a ausência de palpitações sugestivas de taquiarritmia. Também foram considerados como sucesso os casos de fibrilação atrial documentada, ou palpitações taquicárdicas surgidas após a suspensão dos antiarrítmicos, mas que desapareciam com a reintrodução do antiarrítmico.

A ocorrência de fibrilação atrial dentro dos primeiros 45 dias não foi considerada insucesso, pois parece estar ligada ao processo inflamatório produzido pela aplicação de radiofreqüência, não tendo valor prognóstico. Nestes casos, procede-se a reversão e manutenção da medicação antiarrítmica.

A investigação da arritmia, pós-ablação, em cada avaliação do seguimento, foi feita pela análise da história clínica pósablação, eletrocardiograma e Holter de 24 horas (pelo menos dois durante o seguimento).

Os pacientes sintomáticos foram estimulados a comparecer à consulta fora da data agendada, quando foram submetidos a eletrocardiograma e Holter de 24 horas.

$\mathrm{Na}$ avaliação de 3 meses foi procedida a retirada do antiarrítmico de pacientes com ritmo sinusal estável, assintomáticos, com ECG e Holter de 24 horas sem nenhum episódio de fibrilação atrial. O Warfarin foi retirado 6 meses pós-ablação, nos pacientes com sucesso, mas apenas naqueles sem outros fatores de risco para tromboembolismo.

Nos pacientes que se mantiveram em ritmo sinusal, as avaliações subseqüentes consistiram de anamnese, exame físico, ECG e um Holter. Nenhuma medicação antiarrítmica foi introduzida.

A angiorressonância ou angiotomografia foi repetida entre 3 e 6 meses em todos os pacientes.

Não ocorreu a perda de seguimento de qualquer paciente. Todavia, em 5 casos houve comunicação telefônica de que os pacientes teriam apresentado recidiva, sendo atendidos em outros serviços e encaminhados para nova ablação. Esses pacientes foram incluídos no grupo II (insucesso).

O pacote estatístico utilizado foi o SPSS (11.0) sendo utilizados os seguintes testes: Teste $t$ de Student, Teste de Mann-Whitney, Qui-quadrado, Teste exato de Fisher, Análise Multivariada de Cox, Curvas de Kaplan-Meyer - construídas e estratificadas de acordo com as variáveis e comparadas pelo Teste de Log-Rank Curva ROC. Foi considerado o nível de significância de $5 \%=p<0,05$.

\section{Resultados}

Os 104 pacientes foram submetidos a um único procedimento de ablação, que teve tempo médio de $120 \pm 40$ minutos e tempo médio de exposição à radioscopia de $20 \pm 9$ minutos. Dois grupos de pacientes foram identificados durante e ao final do acompanhamento: um grupo considerado com sucesso (Grupo I) compreendendo 87 pacientes e um segundo grupo considerado de insucesso (Grupo II) com 17 pacientes.

A idade média da população amostral foi de 10,9 anos, sendo 72 pacientes $(69,2 \%)$ do sexo masculino e 32 pacientes $(30,8 \%)$ do sexo feminino.

As recorrências ocorreram predominantemente nos primeiros 8 meses. O sucesso total foi de $84 \%$ (fig. 3).

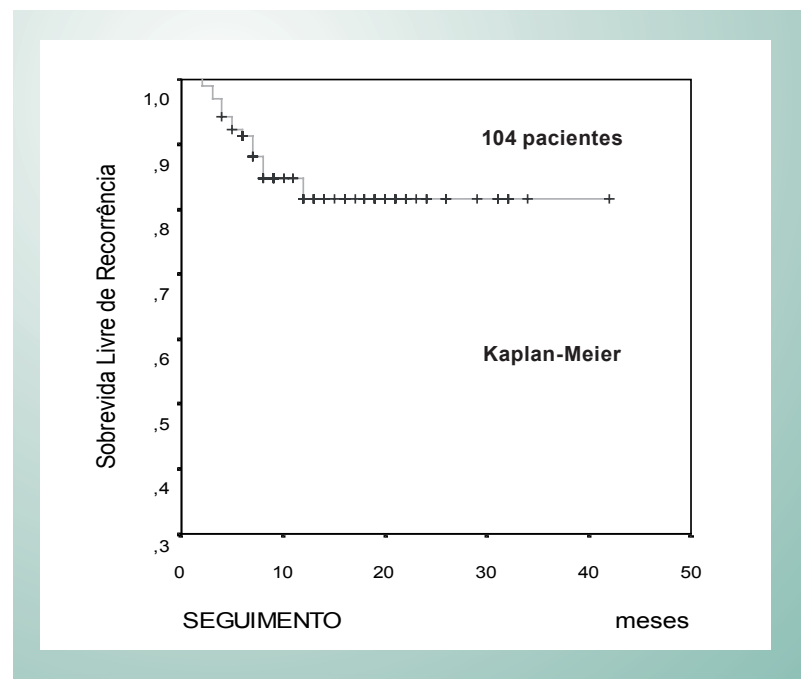

Fig. 3 - Curva de sobrevida livre de eventos da população estudada. Observa-se que a maior parte das recidivas ocorreu nos primeiros 8 meses

Considerando-se a manutenção do ritmo sinusal como objetivo primário observamos dois grupos de pacientes. $\mathrm{O}$ Grupo I (sucesso) foi composto por 87 pacientes, sendo 76 pacientes $(87,4 \%)$ sem recorrência de sintomas ou mesmo arritmias assintomáticas ao Holter, e de um grupo de 11 pacientes $(12,6 \%)$ que apresentaram recorrência da arritmia ou sintomas sugestivos de arritmia, de que tiveram supressão completa com a reintrodução do antiarrítmico.

Ao final do acompanhamento, todos esses pacientes estavam assintomáticos e sem arritmias documentadas.

A presença de palpitação, ainda que sem documentação de fibrilação atrial não responsiva à terapia antiarrítmica, foi considerada critério de insucesso.

O Grupo II (insucesso) compreendeu 17 pacientes que continuaram com fibrilação atrial apesar da tentativa farmacológica de manutenção do ritmo sinusal.

Alguns pacientes foram submetidos a um segundo procedimento ablativo, porém a análise foi feita apenas com os dados da ablação inicial.

Não houve diferença estatística entre os grupos no que concerne à idade e ao sexo. A idade não apresentou distribuição normal, sendo analisada pelas medianas (tab. 1). 


\section{Artigo Original}

\begin{tabular}{|lcc}
\hline Grupos & $\begin{array}{c}\text { Idade } \\
\text { Mediana }\end{array}$ & $\begin{array}{c}\text { Sexo } \\
\mathbf{M} / \mathbf{F}\end{array}$ \\
\hline Sucesso & 59 & $60 / 27$ \\
\hline Insucesso & 61 & $12 / 5$ \\
\hline Geral & 59,5 & $72 / 32$ \\
\hline p & 0,484 & 0,42 \\
\hline Tabela 1 - Distribuição da população amostral, estratificada por \\
grupos, segundo a idade e o sexo
\end{tabular}

A hipertensão arterial sistêmica esteve presente em 56 pacientes $(53,8 \%)$, a hipertrofia ventricular esquerda em 31 pacientes $(29,8 \%)$ e a doença arterial coronariana, em 34 pacientes $(33,6 \%)$. Não houve diferença estatística entre os grupos em relação a essas variáveis (tab. 2).

\begin{tabular}{|c|c|c|c|c|}
\hline Grupos & $\begin{array}{c}\text { HAS } \\
n\end{array}$ & $\begin{array}{c}\text { DAC } \\
n\end{array}$ & $\underset{\mathrm{N}}{\mathrm{HVE}}$ & FE \% \\
\hline $\begin{array}{l}\text { Sucesso } \\
\text { Grupo I }\end{array}$ & 45 & 28 & 27 & 58,68 \\
\hline $\begin{array}{l}\text { Insucesso } \\
\text { Grupo li }\end{array}$ & 11 & 6 & 4 & 56,65 \\
\hline $\mathrm{p}$ & 0,33 & 0,8 & 0,54 & 0,13 \\
\hline \multicolumn{5}{|c|}{$n=$ número de pacientes } \\
\hline \multicolumn{5}{|c|}{$\begin{array}{l}\text { Tabela } 2 \text { - Distribuição da população amostral, } \\
\text { estratificada por grupos, segundo a presença de hipertensão } \\
\text { arterial sistêmica, doença coronariana, fração de ejeção e } \\
\text { hipertrofia ventricular esquerda }\end{array}$} \\
\hline
\end{tabular}

O acompanhamento médio dos pacientes foi de 18 meses, variando de 2 a 42 meses.

A tabela 3 mostra os resultados encontrados quanto ao volume atrial esquerdo, a área de ablação circunferencial direita e esquerda e a completude da linha de radiofreqüência.

Observa-se que o volume atrial esquerdo foi significativamente menor no Grupo I, com sucesso ( $p<$ 0,001).

As áreas ablacionadas foram maiores no grupo sem sucesso, porém este grupo apresentava grandes átrios esquerdos, portanto, esta informação isolada não traduz a verdadeira área em relação à área total.

Mais importante é analisar a área ablacionada em relação ao volume atrial, pois embora uma área circunferencial possa ser idêntica a uma segunda área circunferencial, se um átrio for normal e o outro for aumentado, a área relativa ao átrio de tamanho normal será maior; dito de outra forma, a percentagem de área ablacionada no átrio menor será maior.

A completude das linhas circunferenciais no Grupo I (sucesso) foi obtida em 80/87 pacientes e em 320/348 veias pulmonares. No Grupo II (insucesso) verificou-se que a completude esteve presente em 12/17 significando 51/58 veias. Esta diferença foi estatisticamente significativa com $\mathrm{p}$ $=0,025$ para os pacientes e 0,0038 para as veias. O número de pontos de aplicação de radiofreqüência não diferiu significativamente (tab. 4).

Quando submetidos à análise multivariada, apenas o volume atrial esquerdo $(p<0,0001)$ e a ablação completa $(p<0,05)$ foram preditores independentes de recorrência.

Quando se observa apenas a completude da ablação verifica-se que o grupo com ablação incompleta atingiu apenas $43 \%$ de sucesso e o grupo com linhas completas obteve resultado de $94 \%$ de sucesso ao final do acompanhamento (fig. 4).

O volume atrial esquerdo confirmou ser um importante parâmetro para estratificar os grupos. Quando analisado de forma isolada, apresenta nítida correlação com o prognóstico. Assim, a partir da análise da curva ROC identificou-se o valor de $84 \mathrm{~cm}^{3}$ como corte, identificando os grupos de menor volume como os que mais se beneficiam (fig. 5).

A análise da curva de Kaplan-Meier de sobrevida livre de eventos em pacientes com volume atrial esquerdo maior ou menor que $84 \mathrm{~cm}^{3}$ confirmou que o grupo com átrios esquerdos $<84 \mathrm{~cm}^{3}$ tem maior probabilidade de sucesso ( $p$ $<0,001$ ) (fig. 6)

Quando os dados de volume atrial e completude da ablação circunferencial são associados, pode-se observar que o grupo com volume atrial esquerdo inferior a $84 \mathrm{~cm}^{3} \mathrm{e}$ ablação circunferencial completa atingiu sucesso terapêutico, ou seja, manutenção do ritmo sinusal em 99\% dos pacientes.

\begin{tabular}{|c|c|c|c|}
\hline Variáveis Analisadas & $\begin{array}{l}\text { Sucesso } \\
\mathbf{N}=\mathbf{8 7}\end{array}$ & $\begin{array}{c}\text { Insucesso } \\
\mathbf{N}=17\end{array}$ & $\mathbf{p}$ \\
\hline $\begin{array}{l}\text { Volume AE } \\
\left(\mathrm{cm}^{3}\right)\end{array}$ & $69,50 \pm 21,81$ & $105,10 \pm 20,20$ & $<0,001$ \\
\hline $\begin{array}{l}\text { AVP } \\
\text { Direita }\left(\mathrm{mm}^{2}\right)\end{array}$ & $\begin{array}{c}\text { Mean Rank } \\
49,75\end{array}$ & $\begin{array}{c}\text { Mean Rank } \\
66,59\end{array}$ & 0,035 \\
\hline $\begin{array}{l}\text { AVP } \\
\text { Esquerda }\left(\mathrm{mm}^{2}\right)\end{array}$ & $\begin{array}{c}\text { Mean Rank } \\
46,21\end{array}$ & $\begin{array}{c}\text { Mean Rank } \\
84,68\end{array}$ & $<0,001$ \\
\hline Área D/AE & $0,83 \pm 0,32$ & $0,53 \pm 0,13$ & $<0,001$ \\
\hline Área E/AE & $0,84 \pm 0,33$ & $0,52 \pm 0,18$ & $<0,001$ \\
\hline
\end{tabular}


Artigo Original

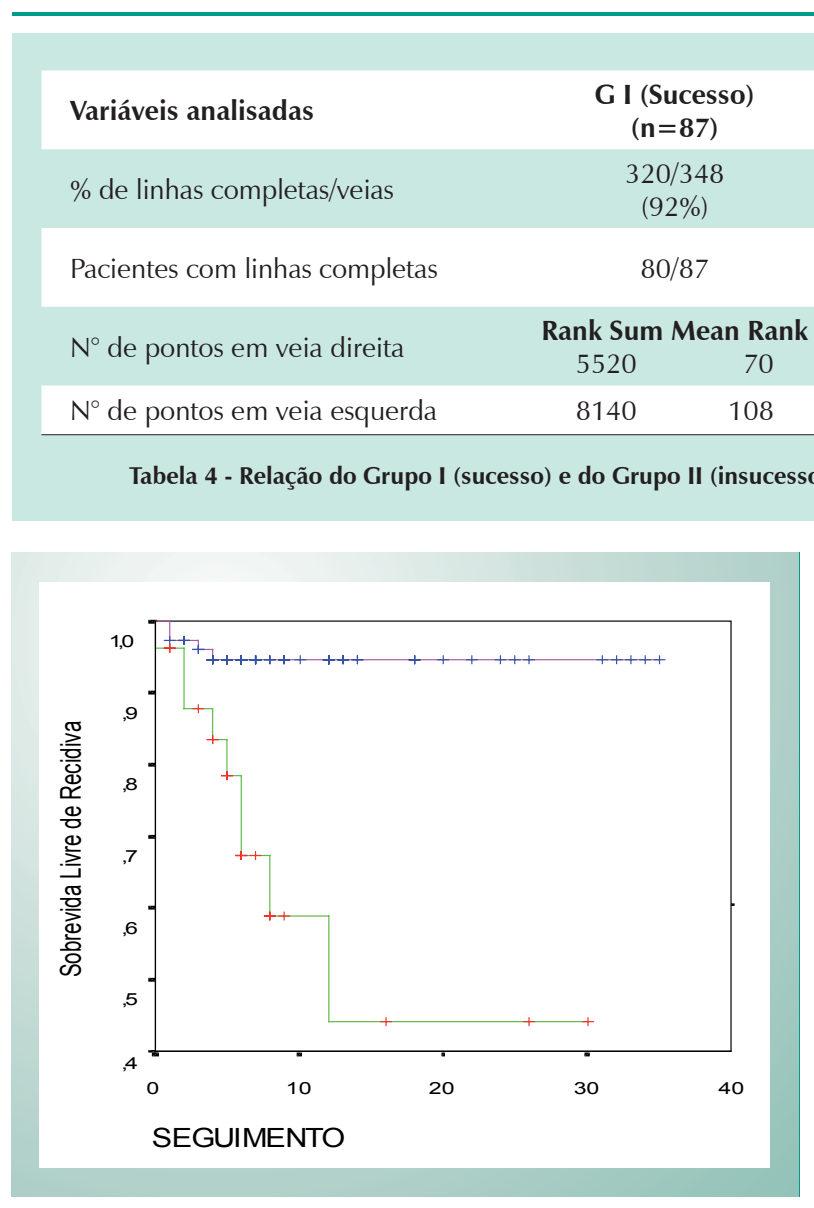

Fig. 4 - Curva de Kaplan-Meier comparando a recidiva entre o grupo com ablação completa e incompleta

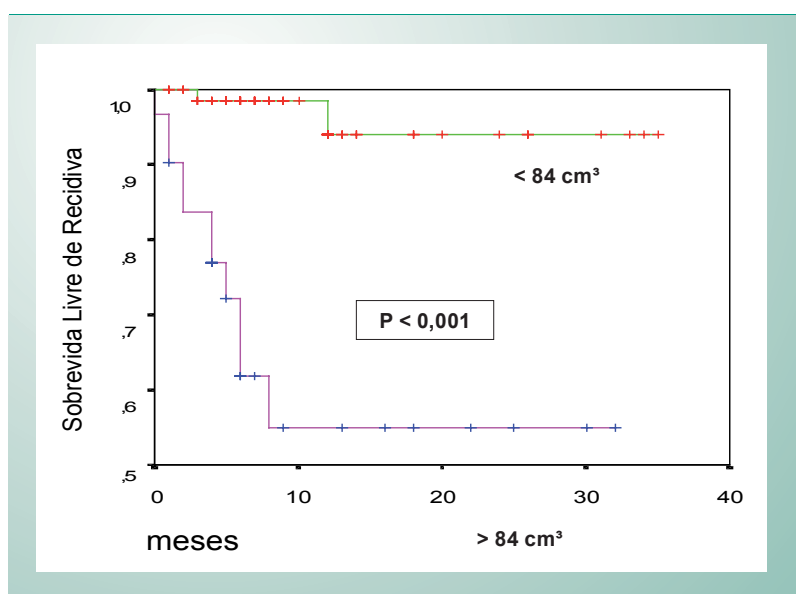

Fig. 6 - Curvas de Kaplan-Meier de sobrevida livre de recidiva estratificada pelo volume atrial esquerdo.

Por outro lado, se ambas as variáveis foram negativas (volume superior a $84 \mathrm{~cm}^{3}$ ) e linha incompleta, o sucesso foi de apenas $34 \%$. A combinação de uma variável positiva com outra negativa, qualquer que fosse, conduziu a um sucesso de $75 \%$ (fig. 7).

Complicações observadas - Dois pacientes apresentaram tamponamento agudo durante a manipulação dentro do átrio esquerdo. Em ambos os casos, fez-se punção pericárdica com

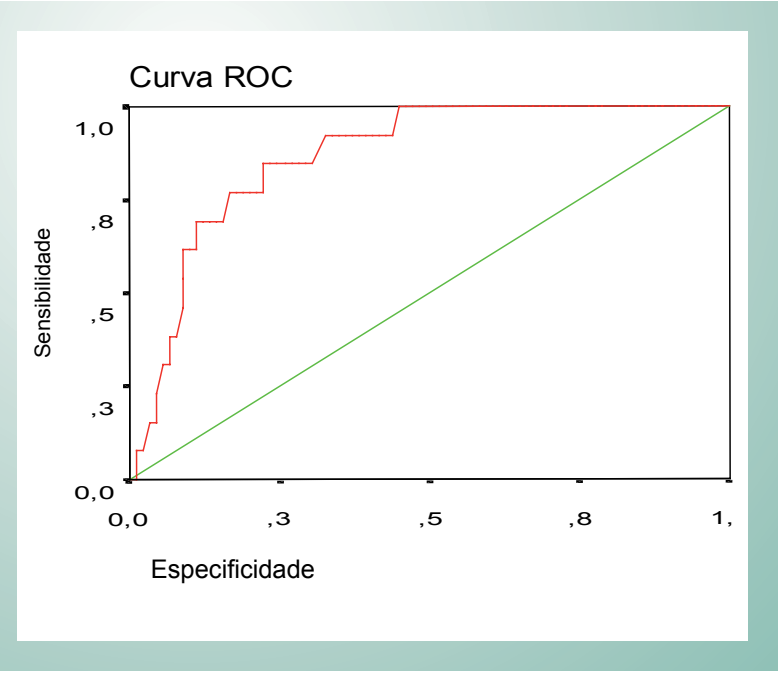

Fig. 5 - Curva ROC.

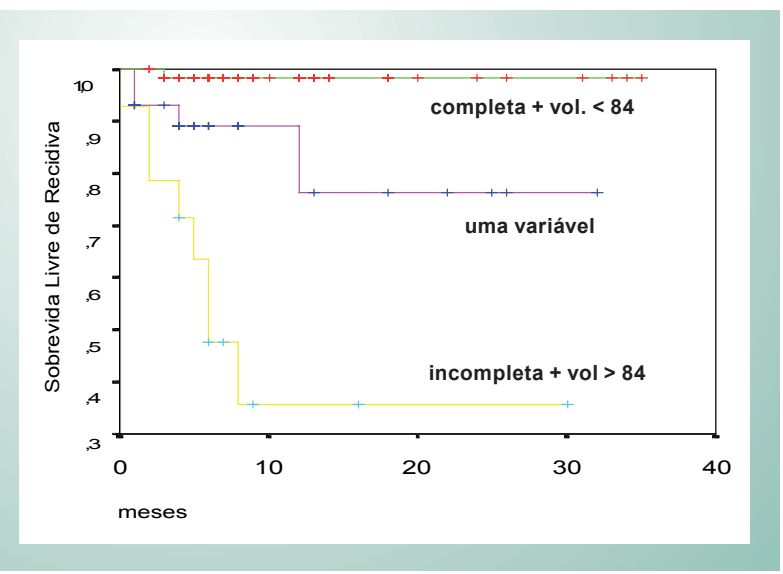

Fig. 7 - Curva de Kaplan-Meier da sobrevida livre de recidiva associando-se o volume e a completude da ablação.

drenagem e interrupção do sangramento ainda no laboratório de eletrofisiologia. Os pacientes obtiveram alta em 72 horas, sem seqüelas clínicas.

Um dos pacientes apresentou tamponamento durante o mapeamento atrial, portanto antes da ablação, sendo interrompido o procedimento. Evoluiu com retorno da fibrilação atrial, tendo sido incluído no grupo de insucesso. Esse mesmo paciente foi reconduzido à ablação em outro 


\section{Artigo Original}

momento, e o procedimento obteve sucesso. Considerando os objetivos deste trabalho, levou-se em conta um único procedimento.

O segundo paciente apresentou tamponamento ao final do procedimento, após a realização da ablação, e evoluiu sem recidiva (sucesso).

Três pacientes apresentaram quadros sugestivos de embolia cerebral de pequena magnitude. Um paciente apresentou discreta alteração da fala; outro, da força do braço direito e outro, da força da mão direita. Todos os quadros reverteram completamente nas primeiras 24 horas, com a manutenção da heparina venosa, não restando qualquer seqüela neurológica nem alteração da ressonância magnética cerebral.

Todos os pacientes foram submetidos a angiotomografia ou angiorressonância de veias pulmonares, entre 3 e 6 meses pós-ablação.

Em 2 pacientes observou-se redução inferior a 30\% no calibre de uma das veias pulmonares, sem clínica. Estes pacientes continuaram evoluindo assintomáticos, não sendo repetido o exame angiotomográfico.

\section{Discussão}

No presente estudo, os 104 pacientes utilizavam drogas antiarrítmicas antes do procedimento, porém sem controle da arritmia. Após a ablação, 87/104 pacientes (84\%) estavam em ritmo sinusal ao final de 18 meses, sendo que apenas 11/87 $(12,6 \%)$ em uso de antiarrítmicos, previamente ineficazes. Nenhuma morte foi observada e nenhum paciente apresentou estenose significativa de veias pulmonares.

Mesmo valendo-se da instrumentalidade do $\mathrm{CARTO}^{\circledR}$, nem todos os grupos apresentam os mesmos resultados. O grupo da Johns Hopkins University School of Medicine (Baltimore, USA) publicou recentemente sua experiência com o mesmo procedimento, apresentando $76 \%$ de sucesso, sendo que $56 \%$ sem antiarrítmicos e $20 \%$ com antiarrítmicos ${ }^{13}$.

Em outro artigo do mesmo grupo relata-se que com uso de ablação por cateter irrigado, com radiofreqüência e isolamento das veias pulmonares associado à linha cavotricuspídea, foi observado sucesso em $65 \%$ dos pacientes (52\% sem antiarrítmicos e $13 \%$ com antiarrítmicos). Para alcançar esse resultado, $14,6 \%$ dos pacientes necessitaram de um segundo procedimento ablativo. Com esta abordagem, apenas a apresentação da fibrilação atrial como persistente, permanente e a idade $>50$ anos foram critérios preditores de recorrência ${ }^{14}$.

Deve-se enfatizar que o sucesso apresentado (84\%) neste estudo refere-se a um único procedimento, o que difere profundamente da desconexão venosa proposta por Haissaguerre, pois, em seu melhor resultado, no qual acrescenta ablação do istmo do átrio esquerdo, com eventual aplicação de radiofreqüência dentro do seio coronário, 32\% dos pacientes necessitaram de um segundo procedimento. Se a desconexão venosa é feita sem bloqueio istmal, 49\% dos pacientes necessitam de um segundo procedimento. Os resultados, com essas elevadas cifras de reintervenção, foram: $87 \%$ de sucesso no grupo com bloqueio istmal e $69 \%$ no grupo sem bloqueio istmal ${ }^{15}$.
Embora localizados nas veias pulmonares, na maioria dos casos, os chamados "gatilhos primários" também podem estar na veia cava superior, no seio coronário, no ligamento de Marshall, ou mesmo em algum ponto do átrio direito ou átrio esquerdo. Por este mesmo motivo, uma terapia que apenas isole as veias pulmonares, logicamente será inferior a outra que englobe parte da musculatura atrial perivenosa, incluindo a desembocadura do ligamento de Marshall.

Os assim chamados "rotores" ou "gatilhos secundários" são áreas de reentrada, ocorrendo primariamente na parede posterior, próximo às veias pulmonares, e possuem um papel crítico na perpetuação da fibrilação atrial ${ }^{16}$. Por isto, a ablação circunferencial não se restringe à abordagem das veias pulmonares.

A melhor técnica para a ablação da fibrilação deve considerar os gatilhos primários, secundários e o substrato atrial. Em estudo randomizado, o grupo de Michigan demonstrou que a ablação atrial esquerda, com linhas que circundam as veias pulmonares (ablação circunferencial), é aproximadamente $20 \%$ mais eficaz que o isolamento das veias pulmonares no tratamento da fibrilação atrial paroxística ${ }^{17}$.

Recentemente, o pioneiro na ablação circunferencial, Carlo Pappone ${ }^{18}$, demonstrou que os pacientes apresentavam menor recorrência quando tinham maior área de ablação, em concordância com os achados deste estudo. Neste mesmo trabalho foi observado que a desnervação vagal, que ocorreu em parte dos pacientes, teve fator adicional protetivo quanto à recorrência de fibrilação atrial.

Sabe-se que $12 \%$ das fibrilações atriais têm na vagotonia seu gatilho indutor e respondem mal à ablação convencional, com desconexão elétrica venosa, ainda que o isolamento seja das 4 principais veias ${ }^{19}$.

Embora não se tenha perseguido a desconexão elétrica venosa, observou-se maior recorrência no grupo de pacientes onde a ablação circunferencial deixou intervalo $>10 \mathrm{~mm}$ entre os pontos contíguos. Isso está de acordo com a teoria multifatorial da fibrilação atrial.

O procedimento puramente anatômico não tem como objetivo desconectar eletricamente as veias pulmonares. Pappone et $\mathrm{a}^{20}$ demonstraram que não houve diferença, usando a ablação circunferencial na freqüência de recorrência, entre os pacientes que apresentavam isolamento ou não.

Mais recentemente, Stabile e cols. (2003), também usando o mapeamento eletroanatômico, relataram ausência da recorrência em $80,4 \%$ dos pacientes, embora somente $40 \%$ destes apresentassem critérios de isolamento elétrico no estudo eletrofisiológico.

A existência de uma descontinuidade $>10 \mathrm{~mm}$, na linha de ablação, correlacionada à maior recorrência é um dado que não identifica a origem da recidiva (dentro das veias ou na região perivenosa). Sabendo-se que tanto as veias como a parede posterior estão envolvidas na gênese e perpetuação da fibrilação atrial e que no procedimento eletroanatômico $\mathrm{o}$ isolamento das veias pulmonares não tem valor preditivo quanto à recorrência, toda a região deve ser encarada como um "complexo" arritmogênico a ser modificado.

A linha de aplicação de radiofreqüência completa não 
só diminui a recorrência de fibrilação atrial como reduz a ocorrência, pós-ablação, de taquicardias atriais iatrogênicas, criadas pelo remodelamento elétrico decorrente das linhas de radiofreqüência ${ }^{21,22}$.

Neste estudo, as ablações circunferenciais mais freqüentemente incompletas foram as relacionadas às veias direitas. Isso se deve à eventual dificuldade de circundar as veias direitas sem perda da punção transeptal. As veias esquerdas estão bem diante do cateter, após a punção transeptal, porém, a discreta parede que separa a aurícula da veia superior esquerda torna as aplicações nessa área trabalhosas.

Do volume atrial - Identificou-se o volume atrial como tendo relação com o resultado final. Esta é apenas mais uma confirmação do que já estava estabelecido: maiores átrios têm mais propensão a arritmias e fibrilação atrial.

Remodelamento é o nome dado ao fenômeno que modifica as características elétricas, geométricas e, finalmente, genéticas do miocárdio atrial.

O remodelamento estende-se através da modulação dos canais de membrana até a modulação genética ${ }^{23}$ que decorre do fato de a perda de atividade contrátil muscular produzir (em minutos ou horas) ajuste genético, resultando em inversão da diferenciação celular, demonstrável pela identificação de supressão do estado diferenciado adulto com "desrepressão" dos genes expressos durante o desenvolvimento - chamada expressão fetoespecífica.

Todas essas modificações são vistas com mais intensidade nos átrios maiores, e certamente isso explica um menor sucesso nos átrios com maior volume, como observado neste estudo. Pappone ${ }^{24}$, valendo-se de medidas ecocardiográficas, observou que havia diferença significativa no diâmetro atrial esquerdo dos pacientes sem recorrência $(40,3 \mathrm{~mm}+4,5 \mathrm{~mm})$ em relação ao grupo que apresentou recorrência $(46,8 \mathrm{~mm}$ $+6,7 \mathrm{~mm}$ ) com $\mathrm{p}<0,001$.

Hakan Oral e cols. ${ }^{17}$, comparando a desconexão venosa e a ablação circunferencial das veias pulmonares, observaram que o tamanho atrial esquerdo foi um preditor independente de recorrência de fibrilação atrial, assim como a ablação circunferencial foi um preditor de melhor resultado.

Assim, fica claro que é importante reverter a fibrilação atrial enquanto o remodelamento não altera significativamente o átrio esquerdo, portanto, na fase de átrio esquerdo normal ou pouco aumentado.

Da área de ablação circunferencial - A área de ablação circunferencial, quando relacionada ao volume atrial, apresentou diferença significativa entre os grupos, na análise univariada. Obviamente um valor absoluto de uma área circunferencial carece de significado, pois os volumes atriais podem variar muito. Dito de outra forma, um valor qualquer de área em um átrio pequeno é muito mais importante que o mesmo valor em um grande volume atrial.

A importância de uma área de ablação que englobe parte da parede posterior é óbvia, considerando-se que, quanto maior for a área abrangida, maior o número de "gatilhos" primários, secundários e circuitos reentrantes isolados do restante do átrio, e menor será a chance de recidiva da fibrilação atrial.

Da análise uni e multivariada - Na análise univariada, o volume atrial esquerdo, a área de ablação circunferencial, a área relacionada ao volume atrial esquerdo e a presença de linhas completas foram preditores de recorrência.

Já na análise multivariada, somente o volume atrial esquerdo e ablação completa foram preditores de recorrência. A curva ROC identificou o ponto de corte de $84 \mathrm{~cm}^{3}$. Assim, os pacientes com volume atrial inferior a $84 \mathrm{~cm}^{3}$ e ablação completa apresentaram elevado nível de sucesso.

Fica clara a necessidade de atuar em átrios não muito aumentados, provavelmente pouco remodelados, e não permitir a existência de gaps na linha de aplicação de energia.

Este último aspecto passa a ter maior relevância exatamente na atualidade, devido às fístulas atrioesofágicas observadas ${ }^{25}$. Com a observância meticulosa da posição do esôfago e as aplicações de radiofreqüência fora da área de contato atrioesofágico, algumas linhas estão sendo realizadas de forma incompleta. É de se esperar que os próximos resultados sejam inferiores aos atuais.

Das complicações - Outro importante aspecto é a ausência de estenoses venosas significativas, observada na população amostral, mesmo com controle da angiorressonância ou angiotomografia. Esta tem sido uma constante nos trabalhos com a ablação circunferencial ${ }^{26}$.

Considerando que apenas $56 \%$ dos pacientes possuem a anatomia clássica de apenas 4 veias pulmonares independentes ${ }^{27}$, a angiotomografia ou angiorressonância usadas são consideradas armas valiosas para evitar aplicação indevida de radiofreqüência em óstio de alguma veia não identificada pela radioscopia, reduzindo-se assim a chance de estenoses venosas.

Embora nenhuma complicação irreversível tenha ocorrido, algumas intercorrências potencialmente graves foram registradas: dois tamponamentos necessitaram de drenagem pericárdica e três acidentes embólicos, tratados clinicamente, não deixaram seqüelas.

A incidência de complicações embólicas na casuística da Cleveland Clinic Foundation foi de 1,5\%, nem todas completamente reversíveis. Tamponamentos também foram observados, chegando a $2 \%$ no grupo de $>60$ anos $^{28}$.

As complicações embólicas estiveram presentes em 3 pacientes neste estudo (2,8\%), ocorrendo de forma transitória, portanto, sem seqüelas. Nos primeiros pacientes da amostra, os tempos de coagulação ativados foram mantidos ao redor de 250, porém, a partir das complicações embólicas, optouse por mantê-los mais próximos de 300 e atualmente até acima deste valor.

Nas séries de Pappone, os tamponamentos foram observados em até $4 \%$ dos pacientes ${ }^{20}$.

Das limitações - Embora estes 104 pacientes fossem intensamente sintomáticos e tenham realizado diversos ECG e Holter de 24 horas, não é possível saber se algum episódio assintomático foi perdido fora dos períodos de monitorização. Sabemos que todo procedimento invasivo pode ter um efeito Placebo imprevisível. 


\section{Artigo Original}

O SOTAP trial que comparou Placebo com Quinidina associada a Verapamil e Sotalol, usou transmissão transtelefônica de 1 minuto diariamente, é o que consideramos mais perto de um acompanhamento perfeito ${ }^{29}$. Mesmo neste caso, a monitorização de 1 minuto por dia significa a nãomonitorização de 1.439 minutos do mesmo dia.

O implante de um monitor de eventos, por 12 meses, para a real avaliação da presença de arritmia incorreria em outros problemas, pois a resposta ventricular em fibrilação atrial com medicação ou doença do nódulo atrioventricular pode ser normal, não deflagrando a marcação dos eventos. Obviamente, os limites éticos impedem tal procedimento em pacientes assintomáticos. Portanto, os sintomas ainda são o critério universalmente aceito para o controle da eficácia do procedimento.

O estudo foi conduzido em um grupo de pacientes selecionados, relativamente jovens, com fibrilação atrial, paroxística e persistente, e função ventricular normal. Estes resultados podem não ser os mesmos em outros grupos, como de pacientes mais idosos, com fibrilação permanente ou disfunção ventricular.

A idade média do grupo amostral foi diferente da idade de maior prevalência de fibrilação atrial na população geral (a população amostral deste estudo foi mais jovem), porém é justamente nesta idade que a fibrilação atrial costuma ser mais sintomática e causar maiores prejuízos na qualidade de vida. Acrescente-se o fato de que indivíduos mais jovens terão mais tempo de exposição às drogas antiarrítmicas, para controle

\section{Referências}

1. Benjamin EJ, Wolf PA, D'Agostinho RB, Silbershatz H, Kannel WB, Levy D. Impact of atrial fibrillation on the risk of death: The Framingham Heart Study. Circulation. 1998; 98: 946-52.

2. Krahn AD, Manfreda J, Tate RB, Mathewson FAL, Cuddy TE. The natural history of atrial fibrillation: incidence, risk factors and prognosis in the Manitoba follow-up study. Am J Med. 1995; 98: 476-84.

3. Psaty BM, Manolio TA, Kuller LH, et al. Incidence of and risk factors for atrial fibrillation in older adults. Circulation. 1997; 96: 2455-61.

4. Vidaillet H, Granada JF, Chyou PH, et al. A population-based study of mortality among patients with atrial fibrillation or flutter. Am J Med. 2002; 113: 365-70.

5. Stewart S, Hart CL, Hole DJ, McMurray JJV. A population-based study of the long-term risks associated with atrial fibrillation: 20-year follow-up of the Renfrew/Paisley study. Am J Med. 2002; 113: 359-64.

6. Go AS, Hylek EM, Phillips KA, et al. Prevalence of diagnosed atrial fibrillation in adults. National implications for rhythm management and stroke prevention: The Anticoagulation and Risk Factors in Atrial Fibrillation (ATRIA) Study. JAMA. 2001; 285: 2370-5.

7. AFFIRM Investigators. A comparison of rate control and rhythm control in patients with atrial fibrillation. N Engl J Med. 2002; 347: 1825-33.

8. AFFIRM Investigators. Relationships between sinus rhythm, treatment, and survival in the atrial fibrillation follow-up investigation of rhythm management (AFFIRM) study. Circulation. 2004; 109: 1509-13.

9. Steinberg JS, Sadaniantz A, Kron J, et al. and the AFFIRM Investigators. Analysis of cause-specific mortality in the atrial fibrillation follow-up investigation of rhythm management (AFFIRM) study. Circulation. 2004; 109: 1973-80.

10. Cappato R, Calkins H, Chen SA, et al. Worldwide survey on the methods, efficacy, and safety of catheter ablation for human atrial fibrillation. Circulation. 2005; 111: 1100-5. do ritmo ou freqüência, o que conduz a maior incidência de efeitos colaterais. E, como a proteção antiarrítmica é incompleta, maior é o risco de eventos embólicos e mais longo o tempo de anticoagulação. Portanto, esta é uma população que tem amplos benefícios do tratamento através da ablação por radiofreqüência.

Os resultados deste estudo foram extraídos do acompanhamento após apenas um único procedimento ablativo. Sabe-se que a maioria dos estudos relata seus resultados com alguns pacientes sendo submetidos a 2 ou 3 procedimentos. A experiência mostra que a repetição da ablação melhora os resultados, porém, o objetivo, neste estudo, foi analisar os resultados de um único procedimento.

Com base nos resultados observados concluímos que a ablação circunferencial da fibrilação atrial, com auxílio do Sistema $\mathrm{CARTO}^{\circledR}$, resultou em manutenção do ritmo sinusal a médio prazo, com um único procedimento, em $84 \%$ dos casos. O volume aumentado do átrio esquerdo, tamanho da área ablacionada e a ablação completa foram preditores de maior recorrência na análise univariada e na análise multivariada, o volume do átrio esquerdo e a ablação completa foram preditores independentes de sucesso do procedimento. Ocorreram 4,8\% de complicações clínicas relevantes.

\section{Potencial Conflito de Interesses}

Declaro não haver conflitos de interesses pertinentes.

11. Nath S, DiMarco J, Haines D. Basic aspects of radiofrequency catheter ablation. J Cardiovasc Electrophysiol. 1994; 5 (10): 863-76.

12. Dorwarth $U$, Fiek $M$, Remp $T$, et al. Radiofrequency catheter ablation; different cooled and noncooled electrode systems induce specific lesion geometries and adverse effect profiles. PACE. 2003; 26 (I): 1438-45

13. Vasamreddy CR, Dalal D, Eldadah Z, etal. Safety and efficacy of circunferential pulmonary vein catheter ablation of atrial fibrillation. Heart Rhythm. 2005; 2: 42-8.

14. Vasamreddy CR, Lickfett L, Jayam VK, et al. Predictors of recurrence following catheter ablation of atrial fibrillation using an irrigated-tip ablation catheter. J Cardiovasc Electrophysiol. 2004; 15 (6): 692-7.

15. Jaïs $\mathrm{P}$, Hocini M, Hsu LF, et al. Technique and results of linear ablation at the mitral isthmus. Circulation. 2004; 110: 2996-3002.

16. Jalife J. Rotors and spiral waves in atrial fibrillation. J Cardiovasc Electrophysiol. 2003; 14: 776-80.

17. Oral H, Scharf C, Chugh A, et al. Catheter ablation for paroxysmal atrial fibrillation: Segmental pulmonary vein ostial ablation vs. left atrial ablation. Circulation. 2003; 108: 2355-60.

18. Pappone C, Santinelli V, Manguso F, et al. Pulmonary vein denervation enhances long-term benefit after circumferential ablation for paroxysmal atrial fibrillation. Circulation. 2004c; 109: 327-34.

19. Oral H, Morady F. Ablation of atrial fibrillation. J Cardiovasc Electrophysiol. 2004; 15: 112-3.

20. Pappone C, Rosanio S, Oreto G, et al. Circumferential radiofrequency ablation of pulmonary vein ostia. A new anatomic approach for curing atrial fibrillation. Circulation. 2000; 102: 2619-28.

21. Pappone C, Rosanio S. Pulmonary vein isolation for atrial fibrillation. In: Douglas Zipes, José Jalife. Cardiac electrophysiology: from cell to bedside. 4. ed. Filadélfia: Elsevier; 2004b: 1039-52. 
22. Cha TJ, Ehrlich JR, Zhang L, Chartier d, Leung TK, Nattel S. Atrial tachycardia remodeling of pulmonary vein cardiomyocytes. Comparison with left atrium and potential relation to arrhythmogenesis. Circulation. 2005; 111:728-35.

23. Pacifico A, Henry PD. Class I or class III agents for atrial fibrillation: Are we asking the right question? PACE. 2003; 26(II): 1613-9.

24. Pappone C, Oreto G, Rosanio S, et al. Atrial electroanatomic remodeling after circumferential radiofrequency pulmonary vein ablation. Efficacy of an anatomic approach in a large cohort of patients with atrial fibrillation. Circulation. 2001; 104: 2539-44.

25. Pappone C, Oral H, Santinelli V, et al. Atrio-esofageal fistula as a complication of percutaneous transcatheter ablation of atrial fibrillation. Circulation. 2004a; 109: 2724-6.
26. Ellenbogen KA, Wood MA. Ablation of atrial fibrillation: Awaiting the new paradigm. J Am Coll Cardiol. 2003; 42 (2): 198-200.

27. Mansour M, Holmvang G, Sosnovik D, et al. Assessment of pulmonary vein anatomic variability by magnetic resonance imaging: implications for catheter ablation techniques for atrial fibrillation. J Cardiovasc Electrophysiol. 2004; 15: 387-93.

28. Bhargava M, Marrouche N, Martin DO et al. Impact of age on the outcome of pulmonary vein isolation for atrial fibrillation using circular mapping technique and cooled-tip ablation catheter: a retrospective analysis. J Cardiovasc Electrophysiol. 2004; 15: 8-13.

29. Patten M, Maas R, Bauer P, Lüderitz B, Sonntag F, Dluzniewski M, et al. for the SOTAP Investigators. Suppression of paroxysmal atrial tachyarrhythmias - results of the SOTAP trial. Eur Heart J. 2004; 25:1395-1404. 